\title{
Aplicação da Oscilação Magnética Controlada ao Arco GMAW Seguindo Padrões de Lissajous Como Forma de Tecimento do Cordão para Promover Distribuição Orientada de Calor e Massa Durante o Processo de Soldagem
}

\author{
Luciano Juliani ${ }^{1}$ Alexandre Queiroz Bracarense ${ }^{2}$, Paulo José Modenesi ${ }^{3}$ (1) \\ ${ }^{1}$ Universidade Federal de Minas Gerais - UFMG, Programa de Pós-Graduação em Engenharia Mecânica - PPGMEC, Belo Horizonte, MG, Brasil. \\ 2 Universidade Federal de Minas Gerais - UFMG, Departamento de Engenharia Mecânica, Belo Horizonte, MG, Brasil. \\ ${ }^{3}$ Universidade Federal de Minas Gerais - UFMG, Departamento de Engenharia Metalúrgica, Belo Horizonte, MG, Brasil.
}

Como citar: Juliani L, Bracarense AQ, Modenesi PJ. Aplicação da oscilação magnética controlada ao arco GMAW seguindo padrões de Lissajous como forma de tecimento do cordão para promover distribuição orientada de calor e massa durante o processo de soldagem. Soldagem \& Inspeção. 2020;25:e2517. https://doi.org/10.1590/0104-9224/SI25.17

\begin{abstract}
Resumo: A deflexão do arco por campos magnéticos permite realizar movimento de tecimento do cordão durante a soldagem utilizando sistemas mecanizados ou automáticos de deslocamento sem a necessidade de empregar dispositivos mecânicos de oscilação. Esta oscilação mecânica já é usada em alguns equipamentos comerciais, principalmente no processo GTAW. O presente trabalho descreve uma nova forma de aplicação da deflexão magnética controlada e sua potencialidade quanto à utilização industrial para promover movimentos complexos de tecimento passando este a seguir padrões paramétricos e matematicamente definidos, conhecidos como figuras de Lissajous. Este recurso pode ser uma boa alternativa quanto ao controle, repetitividade e produtividade para os processos de soldagem. Testes foram realizados nos processos GTAW e GMAW, este último com transferências por curto circuito e pulsada. Os efeitos mais evidentes da oscilação controlada do arco se deram em função do comprimento do arco, amplitude, frequência e formação de uma poça de fusão com maior ou menor volume, característicos de cada processo. No GTAW, os efeitos de tecimento foram obtidos aplicando-se os menores valores de campo magnético. Na soldagem GMAW com transferência por curto circuito, os resultados foram significativos, porém, menos evidentes. No GMAW pulsado, foi possível obter efeitos expressivos de tecimento.
\end{abstract}

Palavra-chave: Tecimento na soldagem; GMAW pulsado; Oscilação do arco; Figuras de Lissajous.

\section{Application of Controlled Magnetic Oscillation to the GMAW Arc, Following Lissajous Patterns as a Weaving Forming the Bead, to Promote Oriented Distribution of Heat and Mass During the Welding Process}

\begin{abstract}
Arc deflection by magnetic fields allows to do weld beads weaving movements in mechanized and automatic systems without mechanical devices of oscillation. The mechanic weaving systems already used in some commercial devices mainly for GTA welding. The present paper describes a new way to apply the magnetic arc deflection that can potentially the industrial application to promote the complex weaving by means parametric and mathematics patterns of Lissajous figures. This feature can be a good option as to control, repeatability and productivity to the welding process. Tests have been done use the GTAW and GMAW processes, the latter with short circuit and pulsed-arc transfer modes. The most apparent effects of controlled arc oscillation occurred to the arc length, range, frequency and molten pool with large or small volume characteristic of each process. On GTAW welding the weaving patterns could be obtained to applying lower magnetic field. With short-circuit GMA welding the results were significant but not very evident. On GMA pulsed welding, was obtained great weavings beads.
\end{abstract}

Key-words: Weaving on welding; GMAW pulsed; Arc oscillation; Lissajous pattern.

\section{Introdução}

Na soldagem a arco, esse atua como uma fonte concentrada de energia capaz de fundir localmente as peças sendo unidas, criando e mantendo uma poça de fusão responsável pela formação do cordão de solda. Tal energia concentrada, se não for corretamente controlada, pode resultar em consequências indesejáveis, como por exemplo, a perfuração da raiz da junta ou a 
degradação de suas propriedades. Executar o tecimento [1-3] durante a soldagem pode ser uma forma eficiente de controlar a distribuição de energia atuante no processo.

A soldagem frequentemente é executada de forma manual ou semiautomática e, para a obtenção de uma junta que atenda às exigências requeridas, existe a necessidade do emprego de um profissional treinado, o que acarreta, muitas vezes, em alto custo da mão de obra. Quando o uso do tecimento é necessário, o soldador executa manualmente movimentos elaborados de acordo com as condições de soldagem e, muitas vezes, com base em sua experiência pessoal.

Por outro lado, falta de ergonomia, fadiga e periculosidade do serviço, baixa produtividade, repetitividade e a decrescente disponibilidade de soldadores bem qualificados, particularmente nos países desenvolvidos, contribuem para o aumento expressivo de operações de soldagem mecanizadas ou automáticas. Para realizar o tecimento, com essas formas de operação e em particular com os processos GMAW e GTAW, podem ser usados dispositivos mecânicos [4-8] ou aplicados campos magnéticos capazes de defletir o arco elétrico [9-16]. Geralmente, por meio de fluxo magnético o arco é movimentado ortogonalmente ao sentido de soldagem [9-12], para frente e para trás [13-15] ou de forma circular [16]. No caso do tecimento ser feito por meios mecânicos é mais comum os equipamentos produzirem deflexões ortogonais à soldagem, mas também podem produzir formas circulares [17]. Sejam os tecimentos, produzidos por meios magnéticos ou mecânicos, ambos apresentam algumas limitações quanto ao uso, por exemplo: histerese para deflexões magnéticas de alta frequência [18-20] e dificuldade de acesso em soldagem de chanfro profundo para os mecânicos [21].

Por outro lado, dispositivos magnéticos apresentam potencial para a realização de padrões elaborados de deflexão e manipulação do arco quando mais de um conjunto de eletroímãs são usados [14,16]. Computacionalmente, padrões similares aos tecimentos da soldagem manual (ou semiautomática) podem ser reproduzidos em soldagem mecanizada e automática usando figuras de Lissajous. Essas figuras são curvas paramétricas matematicamente traçadas em um plano por pontos $\{x(t)$, $y(t)\}$ determinados por equações do tipo senoidal [22]:

$x(t)=A_{x} \operatorname{sen}\left(2 \pi F_{x} t+\phi\right)+O_{x}$

$y(t)=A_{y} \operatorname{sen}\left(2 \pi F_{y} t\right)+O_{y}$

onde $A_{x}$ e $A_{y}$ são as amplitudes da curva nas direções x e y, respectivamente, $F_{x}$ e $F_{y}$ são as frequências das funções geradores, $\phi$ é a ângulo de fase entre elas (em radianos) e $O_{x}$ e $O_{y}$ são os offsets. Variando esses parâmetros, diferentes curvas podem ser geradas. Por exemplo, quando $F_{x}=F_{y}$ e $\phi=\pi / 2$, são geradas elipses e, em particular, se $A_{x}=A_{y}$, um círculo é gerado. Exemplos adicionais de figuras de Lissajous são mostrados na Figura 1. Observa-se, ainda, que, substituindo as senoides (Equação 1 e Equação 2) por outras funções periódicas, novas curvas podem ser obtidas.

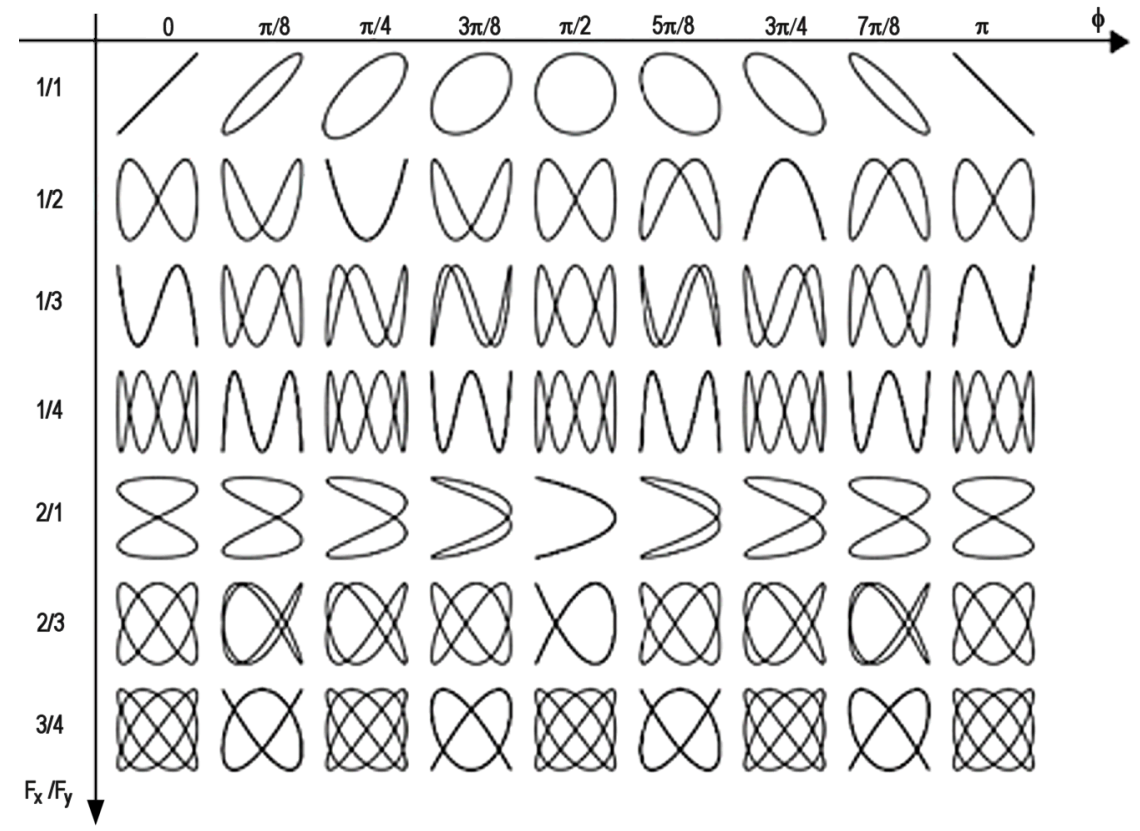

Figura 1. Exemplos de padrões de figuras de Lissajous em função das razões entre as frequências $\left(F_{x} / F_{y}\right)$ e o ângulo de fase $(\phi)$. 
Buscando implementar padrões de tecimento em soldagens, tais que reproduzam figuras de Lissajous em processos mecanizados ou automáticos, foi desenvolvido no Laboratório de Robótica, Soldagem e Simulação (LRSS) da Universidade Federal de Minas Gerais um sistema que utiliza dois eletroímãs dispostos nas direções transversal e longitudinal ao sentido de soldagem. Estes podem ser alimentados por tensões com diferentes padrões de onda. O presente artigo descreve, basicamente sobre o desenvolvimento deste sistema e, de forma mais enfática, apresenta sua utilização prática no sentido de promover melhorias quanto à distribuição de calor e massa fundida na região da soldagem e ao entorno dela, objetivando conseguir um cordão de solda adequado capaz de atender às exigências de uma junta. Com isso acredita-se ser possível alcançar maior qualidade, produtividade e condições de trabalho mais favoráveis para os processos de soldagem.

\section{Metodologia}

Para a movimentação controlada do arco foi projetado e construído um equipamento constituído por fontes controladoras da tensão elétrica que alimentam bobinas de um par de eletroímãs para a oscilação do arco. Os dois eletroímãs são montados em um dispositivo (adaptador) que permite a sua instalação em tochas para soldagem. Com este sistema é possível produzir campos magnéticos variáveis que podem atuar no arco elétrico de forma a fazer com que estes possa ser defletido seguindo padrões de figuras de Lissajous.

O módulo de potência e controle desse sistema permite alimentar cada eletroímã com tensão variável segundo ondas de formato senoidal, triangular ou quadrada. O módulo permite especificar a frequência das ondas (de 0,1 até 4,5 Hz), suas amplitudes (até $24 \mathrm{~V}$ ) e ângulo de fase (de zero até $\pi \mathrm{rad}$ ). Além disso, é possível atuar na simetria (de até $90 \%$ em intervalos de $10 \%$ ) e offset (deslocamentos relativos entre os eixos), de até $12 \mathrm{~V}$ em cada eixo. É importante salientar que cada uma desses parâmetros pode ser alterado independentemente para cada eixo.

Em princípio, os exemplos de padrões de Lissajous mostrados na Figura 1 podem ser produzidos com o sistema desenvolvido. Entre esses, serão usados nos testes realizados no presente trabalho os padrões correspondentes a valores de razão de frequências $\left(F_{x} / F_{y}\right)$ e ângulo de fase $(\phi)$ iguais a $1 / 1-\pi / 2 ; 1 / 2$ - 0 e $1 / 2-\pi / 4$ (Figura 1 ). Além desses, um padrão especial obtido pela combinação de uma onda quadrada no eixo " $X$ " e uma triangular no " $Y$ " com $F_{x} / F_{y}$ igual a $1 / 2$ e $\phi=\pi / 4$, Figura 2 , também foi explorado. Um osciloscópio do fabricante TEKTRONIX, modelo TPS 2024B, foi ligado às saídas do módulo de potência e controle antes dos testes de soldagem e usado para confirmar o padrão de tecimento gerado.

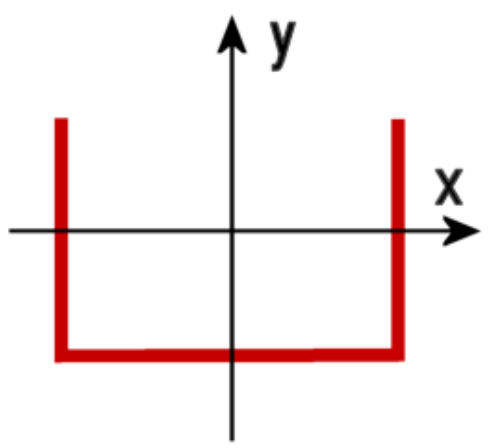

Figura 2. Figura (denominada de "deslocamento lateral") obtida com aplicação de uma onda quadrada no eixo " $X$ " e de uma onda triangular no eixo " $y$ ".

Na Figura 3, é mostrado o dispositivo com os dois eletroímãs geradores/direcionadores de fluxo magnético montados em uma tocha para soldagem GMAW. Cada eletroímã é excitado por um conjunto de duas bobinas (geradores) que podem ser alimentadas em conjunto ou separadamente permitindo variações do campo magnético. Os direcionadores de fluxo são os núcleos das bobinas que, para o presente estudo, foram construídos com aço baixo carbono, ASTM A36.

Neste trabalho, a parte experimental foi desenvolvido da seguinte forma:

- $\quad$ Calibração do campo magnético gerado pelo sistema;

- Testes sobre chapa utilizando o processo GTAW autógeno;

- $\quad$ Teste sobre chapa com o processo GMAW com transferências por curto circuito e pulsada. 


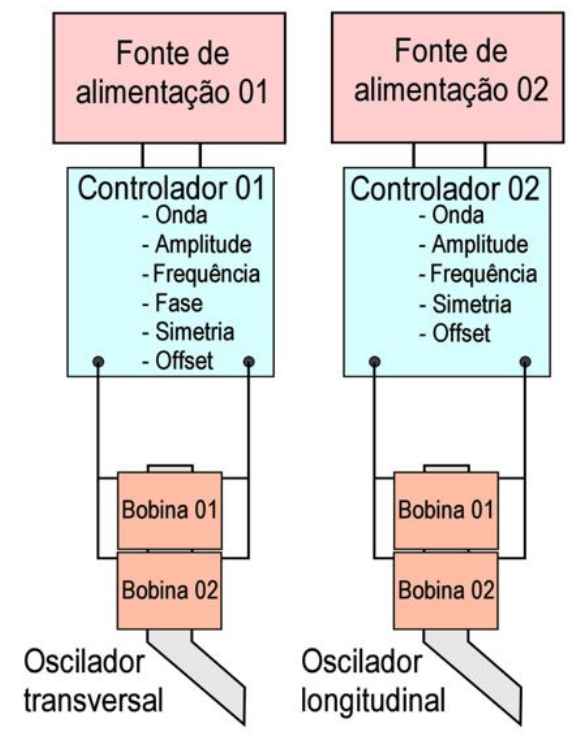

(A)

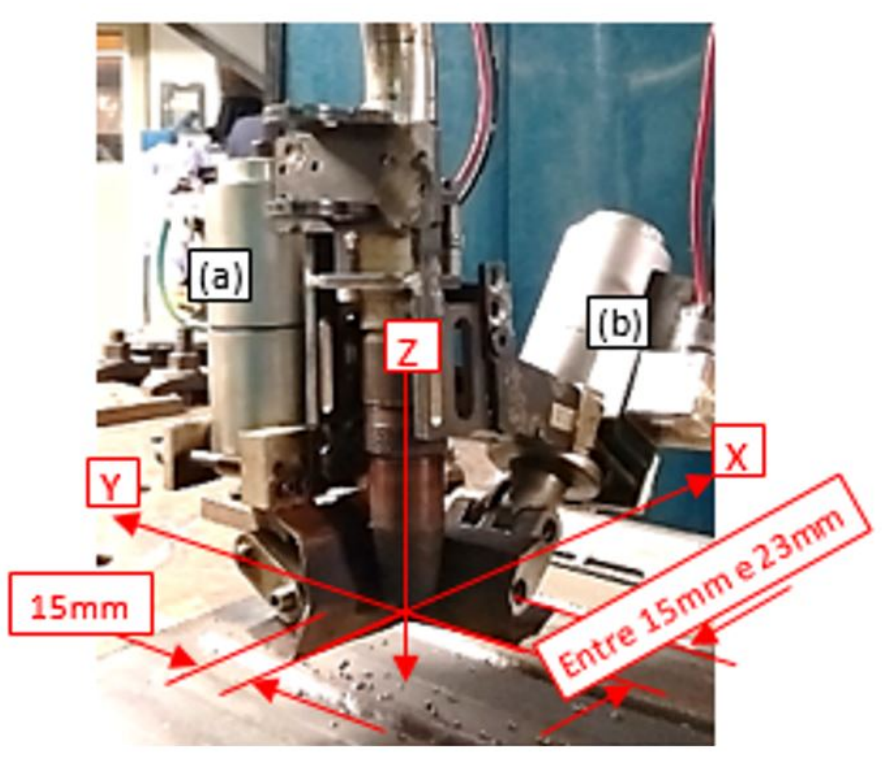

(B)

Figura 3. (A) Diagrama do sistema de oscilação magnética do arco; (B) Osciladores (eletroímãs) montados na tocha GMAW: (a) bobinas responsáveis pela oscilação no eixo " $\mathrm{X}$ " e (b) bobinas responsáveis pela oscilação no eixo " $Y$ ". O eixo " $Z$ " refere-se à posição do arame eletrodo.

Para a calibração do sistema, um medidor de densidade de fluxo magnético (Teslâmetro) do fabricante PHYWE, com 3 faixas de medições (entre 0-20mT; 0-200mT e 0-2000mT) foi usado para medir as densidades de campo magnético produzidos por cada eletroímã com diferentes níveis de alimentação em suas bobinas. As medições foram feitas posicionando-se a ponta de prova do Teslâmetro a $15 \mathrm{~mm}$ de distância da ponta do núcleo de cada eletroímã conforme é mostrado na Figura 4. Distância similar foi usada entre os eletroímãs e o arame nos testes com o processo GMAW. Nos testes com o processo GTAW, os eletroímãs foram posicionados a uma distância de $23 \mathrm{~mm}$ do eletrodo no sentido do eixo " $\mathrm{X}$ " e de $15 \mathrm{~mm}$ no sentido do eixo " $\mathrm{Y}$ ".

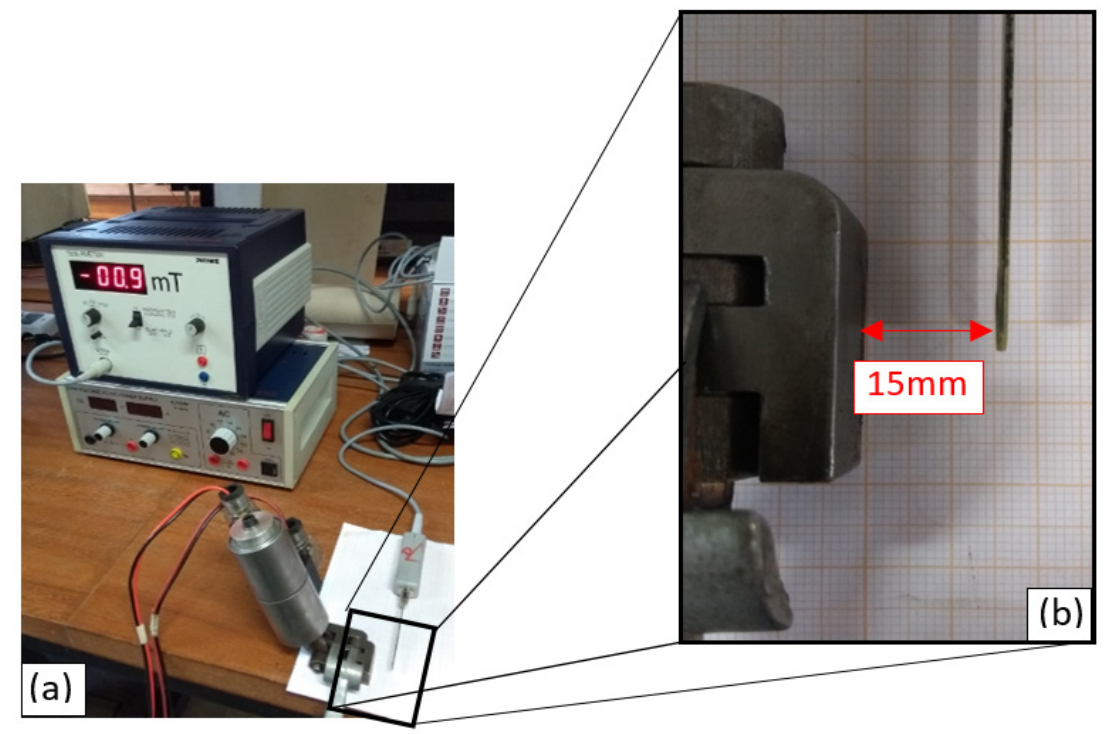

Figura 4. Bancada de testes para a medição da densidade de fluxo. (a) Medidor de densidade de fluxo magnético (Teslâmetro); (b) ampliação da região de medição.

A tensão de alimentação das bobinas dos eletroímãs foi verificada comparando-a com as tensões fornecidas por uma fonte variável de dois canais, modelo SF 9584 A, do fabricante PASCO.

Todos os testes de soldagem foram realizados sobre corpos de prova feitos de chapa de aço carbono ASTM A36 com largura de $100 \pm 5 \mathrm{~mm}$, comprimento de $230 \pm 5 \mathrm{~mm}$ e espessura de 5,0 $\pm 0,5 \mathrm{~mm}$. Todas as soldas foram produzidas na posição plana. 
As soldagens GTAW foram realizados de forma autógena. Esses testes tiveram como objetivo avaliar a capacidade do sistema de oscilar o arco em condições simples, isto é, sem transferência de metal e com a distância da ponta do eletrodo à peça mantida constante. Foi empregada uma fonte com controle eletrônico, modelo Master TIG 2500W do fabricante KEMPPI, proteção de argônio puro com vazão de $10 \pm 0,5 \mathrm{~L} / \mathrm{min}$, eletrodo de tungstênio puro com diâmetro de $2,4 \mathrm{~mm}$ e bocal cerâmico $\mathrm{n}^{\circ}$ 10. Trabalhou-se com corrente de $120 \mathrm{~A}$, distância entre a ponta do eletrodo e o metal base de $7 \pm 1 \mathrm{~mm}$ e uma velocidade de soldagem de $1,4 \mathrm{~mm} / \mathrm{s}$. Os padrões de oscilação testados são mostrados na Tabela 1 . Nessa tabela e nas demais, os valores de amplitude (em $\mathrm{mT}$ ) foram calculados a partir dos valores de tensão selecionados no sistema de oscilação do arco usando as Equações 3 e 4 apresentadas na próxima seção.

Tabela 1. Parâmetros de oscilação utilizados para a soldagem GTAW autógena.

\begin{tabular}{|c|c|c|c|c|}
\hline & \multicolumn{2}{|c|}{ Condição (a) } & \multicolumn{2}{|c|}{ Condição (b) } \\
\hline & “X” & "Y" & "X" & "Y" \\
\hline Tipo de onda & Seno & Seno & Seno & Seno \\
\hline Amplitude (mT) & 9,9 & 7,9 & 9,9 & 2,8 \\
\hline Frequência (Hz) & 0,5 & 1,0 & 0,5 & 0,5 \\
\hline Simetria (\%) & 50 & 50 & 50 & 50 \\
\hline Offset & 0 & 0 & 0 & 0 \\
\hline Ângulo de fase (rad) & $\pi / 4$ & --- & $\pi / 2$ & --- \\
\hline
\end{tabular}

Obs.: "X" = Oscilação transversal. "Y" = Oscilação longitudinal.

Os testes de soldagem GMAW com transferência por curto circuito foram realizados utilizando uma fonte de tensão constante, modelo MotoArc 450 do fabricante MOTOMAN com arame AWS ER70S6 com diâmetro de 1,2 mm e proteção de $\mathrm{Ar}-20 \% \mathrm{CO}_{2}$ a $15 \mathrm{~L} / \mathrm{min}$. Estes testes, objetivaram avaliar o desempenho do sistema de oscilação na presença de transferência de metal e com comprimento de arco variável (em média, pequeno). Caracteristicamente, na transferência por curto circuito, o comprimento do arco varia constantemente desde sua completa extinção, por causa da transferência metálica que acontece devido ao efeito da tensão superficial quando a gota/arame toca a peça (curto circuito), até seu comprimento máximo, o qual varia constantemente devido à recuperação gradual da distância entre a ponta do eletrodo e a peça, velocidade de fusão do metal na ponta do arame e também da velocidade de alimentação deste que, por sua vez, é controlada pelo equipamento de soldagem.

Diversos testes foram realizados, mas, no presente trabalho, serão apresentados os resultados relativos a testes com uma distância do bico de contato à peça (DBCP) de $18 \mathrm{~mm}$, tensão e correntes médias de operação de aproximadamente $18 \mathrm{~V}$ e $165 \mathrm{~A}$, respectivamente e velocidade de soldagem de $4,2 \mathrm{~mm} / \mathrm{s}$, pois, apresentaram boa estabilidade tanto para os testes sem oscilação quanto para aqueles onde, o único padrão de oscilação usado, está mostrado na Tabela 2.

Tabela 2. Parâmetros de oscilação utilizados para os testes com o processo GMAW com transferência por curto-circuito.

\begin{tabular}{|c|c|c|}
\hline & “X” & "Y" \\
\hline Tipo de onda & Seno & Seno \\
\hline Amplitude (mT) & 12,9 & 5,6 \\
\hline Frequência (Hz) & 0,5 & 0,5 \\
\hline Simetria (\%) & 50 & 50 \\
\hline Offset & 0 & 0 \\
\hline Ângulo de fase (rad) & $\pi / 2$ & --- \\
\hline
\end{tabular}

Obs.: "X" = Oscilação transversal. "Y" = Oscilação longitudinal.

Nos testes de soldagem GMAW pulsada, foi utilizada uma fonte com controle eletrônico, modelo MTE DIGITEC 600 do fabricante IMC, na configuração para imposição de corrente, com proteção de $\mathrm{Ar}-4 \% \mathrm{CO}_{2}$ e o mesmo arame que foi usado anteriormente. Os testes neste modo de transferência objetivaram analisar a transferência metálica mantendo-se um comprimento de arco relativamente constante. A expressão "relativamente constante" refere-se às ocorrências de pequenas flutuações no comprimento do arco devido às incursões periódicas entre os parâmetros de pico e de base e a influência do campo magnético que atua no arco promovendo sua deflexão. As condições de soldagem usadas, levantadas após uma série de testes exploratórios, estão indicadas na Tabela 3. Com essas condições obteve-se um comprimento do arco de 
aproximadamente $5 \mathrm{~mm}$, medido conforme a Figura 5 em testes sem aplicação de oscilação magnética e utilizando a técnica de superposição de imagens por meio do software VEGAS Pro 15.0.

Tabela 3. Parâmetros utilizados nos testes com soldagem GMAW pulsada.

\begin{tabular}{cc}
\hline Corrente de pico $[\mathrm{A}]$ & 292 \\
Corrente de base $[\mathrm{A}]$ & 87 \\
Tempo de pico $[\mathrm{ms}]$ & 3,5 \\
Tempo de base $[\mathrm{ms}]$ & 13,6 \\
Vazão do gás $[\mathrm{L} / \mathrm{min}]$ & 18 \\
Veloc. Alimen. de arame $[\mathrm{m} / \mathrm{min}]$ & 3,5 \\
Veloc. Soldagem $[\mathrm{mm} / \mathrm{s}]$ & 4 \\
DBCP $[\mathrm{mm}]$ & 18 \\
\hline
\end{tabular}

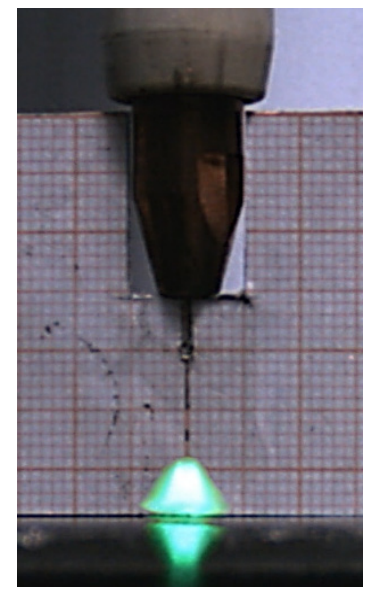

Figura 5. Medição do comprimento do arco no processo GMAW pulsada.

As condições de oscilação usadas estão na Tabela 4. Observar que, na condição (e), a frequência de oscilação do arco foi muito inferior ao usualmente usado em soldagem. Assim, nessa condição, o deslocamento do arco pelo campo magnético ocorreu de uma forma relativamente lenta em comparação com as demais. Adicionalmente, foi feito mais um teste em que o arco foi oscilado apenas transversalmente com um campo magnético de 7,4 mT usando uma onda quadrada com frequência de $0,1 \mathrm{~Hz}$, condição (g). Assim, as condições (f) e (g) objetivaram avaliar efeitos dinâmicos da oscilação magnética relacionados com a formação do cordão na soldagem GMAW pulsada.

Tabela 4. Parâmetros de oscilação utilizados nos testes com soldagem GMAW pulsada.

\begin{tabular}{|c|c|c|c|c|c|c|c|c|c|c|}
\hline & \multicolumn{2}{|c|}{ Condição (c) } & \multicolumn{2}{|c|}{ Condição (d) } & \multicolumn{2}{|c|}{ Condição (e) } & \multicolumn{2}{|c|}{ Condição (f) } & \multicolumn{2}{|c|}{ Condição (g) } \\
\hline & "X" & "Y" & "X" & "Y" & "X" & "Y" & "X" & "Y" & "X" & "Y" \\
\hline Tipo de onda & Seno & Seno & Seno & Seno & Seno & Seno & Seno & Seno & Quadr. & Triang \\
\hline Amplitude (mT) & 5,6 & 3,0 & 11,6 & 6,8 & 8,5 & 8,5 & 8,5 & 8,5 & 7,4 & 0,1 \\
\hline Frequência (Hz) & 0,5 & 1,0 & 0,5 & 1,0 & 0,1 & 0,1 & 0,5 & 0,5 & 0,1 & 0,1 \\
\hline Simetria (\%) & 50 & 50 & 50 & 50 & 50 & 50 & 50 & 50 & 10 & 90 \\
\hline Offset & 0 & 0 & 0 & 0 & 0 & 0 & 0 & 0 & 0 & 0 \\
\hline Ângulo de fase (rad) & $\pi / 4$ & --- & $\pi / 4$ & --- & $\pi / 2$ & --- & $\pi / 2$ & --- & $\pi$ & --- \\
\hline Padrão & \multicolumn{2}{|c|}{ “Meia-Lua” } & \multicolumn{2}{|c|}{ “Meia-Lua” } & \multicolumn{2}{|c|}{ "Círculo" } & \multicolumn{2}{|c|}{ "Círculo" } & \multicolumn{2}{|c|}{ “Desloc. lateral” } \\
\hline
\end{tabular}

Obs.: "X" = Oscilação transversal. "Y" = Oscilação longitudinal.

Para a soldagem GTAW autógena e a GMAW pulsada, um sistema de deslocamento com velocidade constante do fabricante IMC, modelo TARTILOPE V1, foi utilizado para conduzir os corpos de prova ao longo do percurso de trabalho sendo que as respectivas tochas permaneceram fixas. E nas GMAW por curto circuito foi utilizado um robô antropomórfico MOTOMAN modelo SK6 com 6 graus de liberdade, onde, a peça ficou parada e a tocha foi conduzida com velocidade constante. 
As soldas produzidas com o processo GTAW e com o processo GMAW com transferência por curto circuito foram analisadas visualmente buscando-se identificar, na superfície dos cordões, indícios do efeito do movimento imposto ao arco elétrico. Características geométricas nas superfícies do cordão foram medidas, em fotografias desses, com o programa Image J.

Nos testes de soldagem GMAW pulsada, foi feita a aquisição de sinais elétricos com uma taxa de 3,6 kHz usando um sistema modelo TC-1 da IMC. Além dos dados de corrente e tensão de soldagem, foram também medidas as tensões de alimentação das bobinas dos dois eletroímãs. Os arquivos de dados obtidos foram analisados usando o programa SINAL desenvolvido no Laboratório de Robótica, Soldagem e Simulações - LRSS da UFMG.

Na Figura 6 é mostrada uma fotografia da montagem da bancada de testes onde foram efetuadas as soldagens GTAW autógeno e GMAW pulsada, sendo: (a) Mesa de soldagem IMC, (b) respectivas tochas de soldagem, (c) Sistema de deslocamento com velocidade constante, (d) Bobinas.

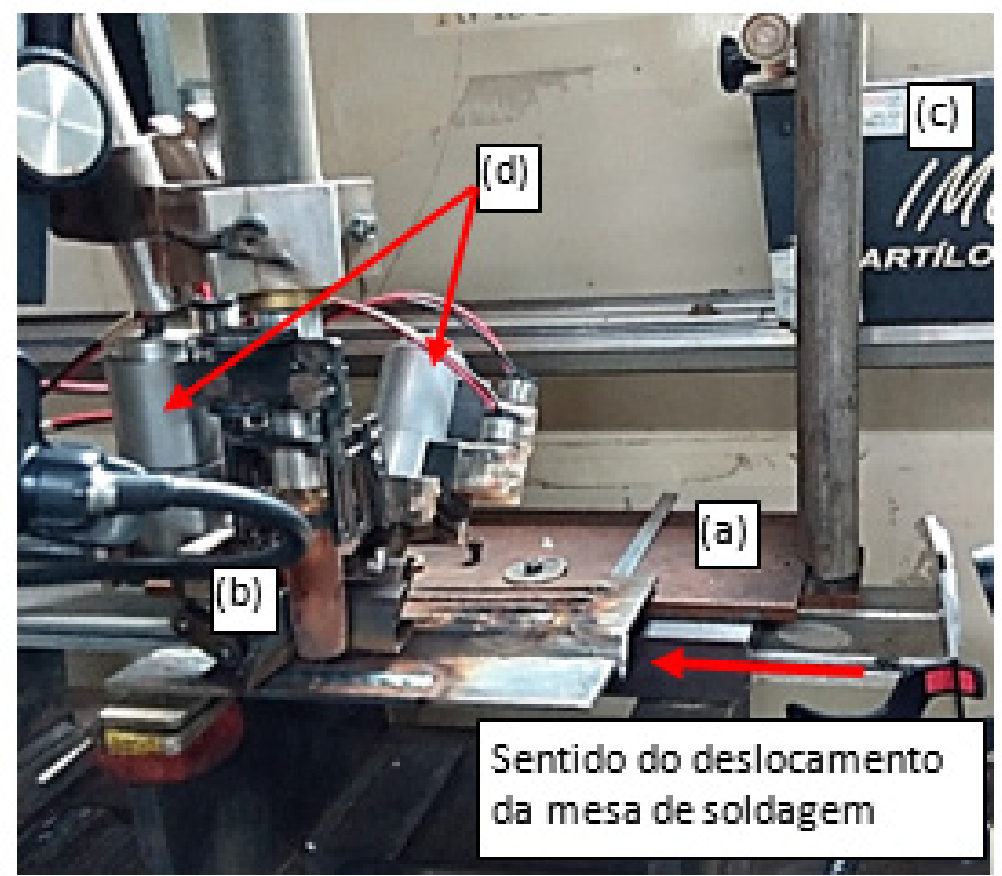

Figura 6. Montagem da bancada de testes.

\section{Resultados e Discussão}

Os resultados das medidas dos campos magnéticos produzidos pelos eletroímãs são mostrados na Figura 7 juntamente com as linhas de tendência obtidas pelas regressões, linear (Equação 3) e polinomial de grau 2 (Equação 4) dos dados. Os modelos de regressão obtidos, respectivamente para alimentação com uma e duas bobinas, foram:

$B=0,1756 \cdot U_{1 B}$

$B=0,7797 \cdot U_{2 B}-9,880 \cdot 10^{-3} U_{2 B}^{2}$

Estas equações foram obtidas por meio do software EXCEL, o qual disponibiliza várias ferramentas de análise estatística e ajustes de curvas formadas a partir dos dados obtidos dos experimentos, onde " $B$ " é o campo magnético estimado (mT) e $U_{1 B}$ e $U_{2 B}$ são as tensões $(V)$ de alimentação com uma e duas bobinas, respectivamente. Observa-se que, no caso da alimentação com duas bobinas, os dados experimentais foram melhor modelados por uma equação polinomial de 20 grau. A falta de linearidade observada nesse caso pode estar ligada à saturação magnética parcial do núcleo de aço baixo carbono usado para a construção dos núcleos dos eletroímãs ou ter sido atingido o limite máximo de geração de campo magnético fornecido pelas bobinas. 

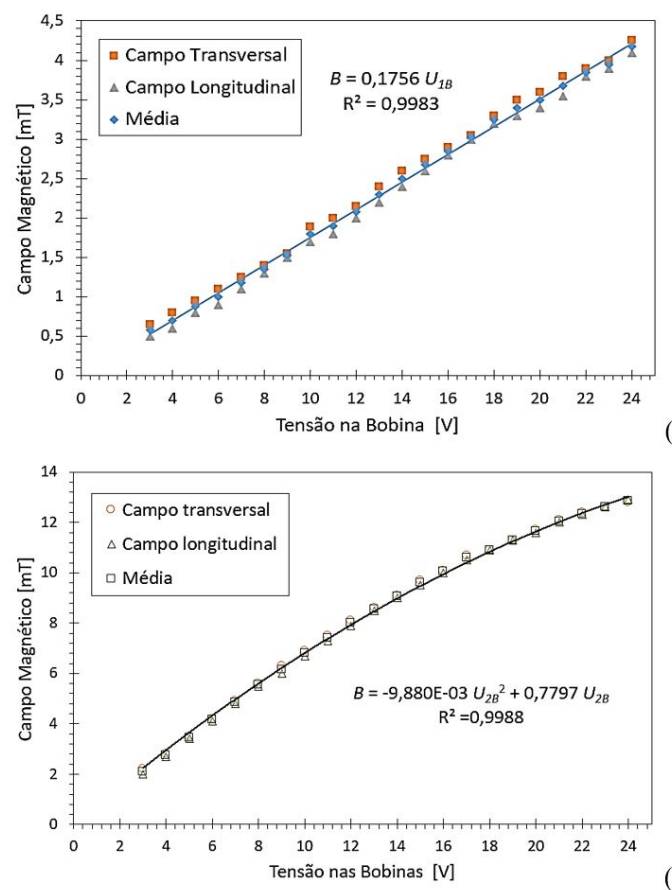

(a)

(b)

Figura 7. Medições das densidades de fluxo magnético produzido pelos eletroímãs do sistema de oscilação do arco. Em (a) alimentação com uma bobina e (b) com duas bobinas.

Na Figura 8 são mostrados exemplos de padrões gerados pelas tensões fornecidas para os osciladores transversal e longitudinal do sistema que foi desenvolvido. Considerando particularmente as condições em que o comportamento dos eletroímãs é linear (Figura 8), espera-se inicialmente que esses padrões de tensão gerem uma indução magnética variável na região do arco que tenderá a defletir o arco segundo padrões similares aos mostrados.

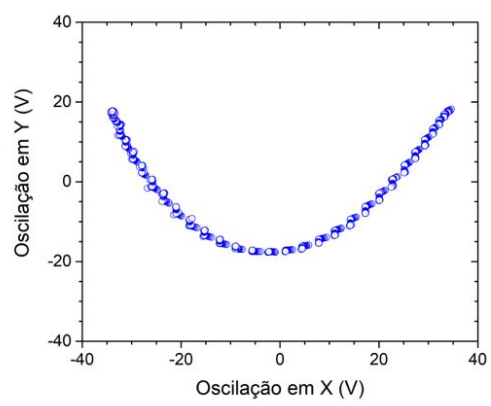

(a)

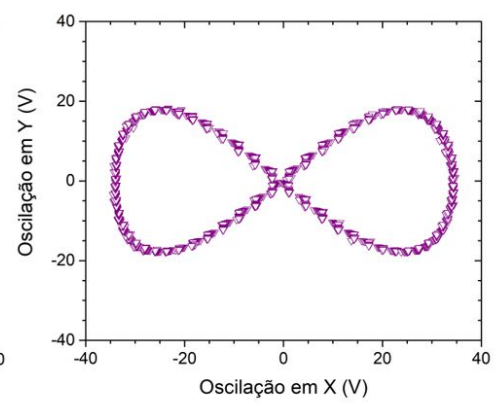

(b)

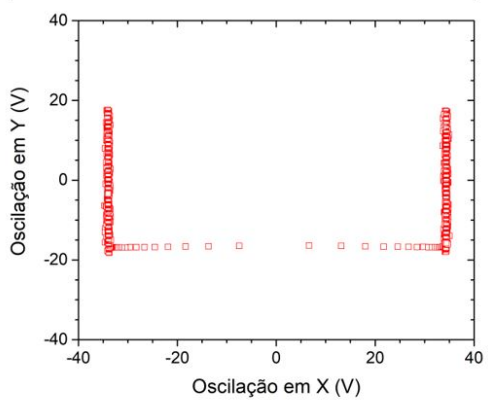

(c)

Figura 8. Exemplos de padrões de oscilação do arco gerados pela alimentação de tensão nas bobinas. (a) “Meia-lua” (Fx/Fy = $1 / 2-\pi / 4$ da Figura 1); (b) "Infinito" (Fx/Fy = 1/2 - $\pi / 2$ da Figura 1); e (c) "Deslocamento lateral" (padrão mostrado na Figura 2). Oscilação em " $X$ " e " $Y$ " referem-se a transversal e longitudinal, respectivamente. 
Dois cordões produzidos com o processo GTAW usando os padrões de tecimento especificados na Tabela 1 são mostrados na Figura 9.

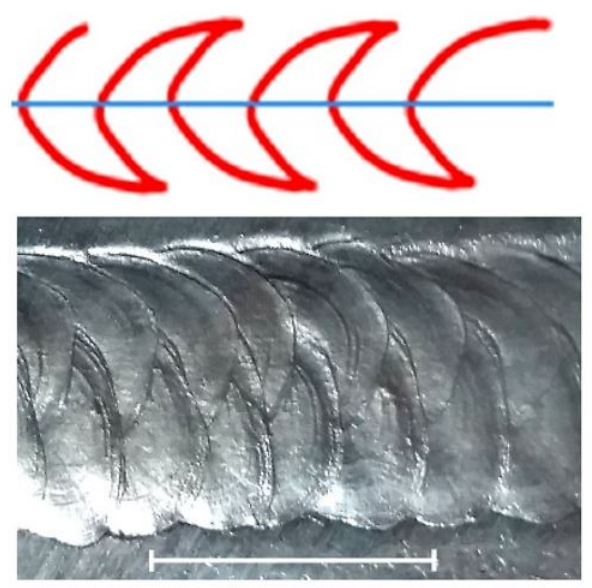

(a)
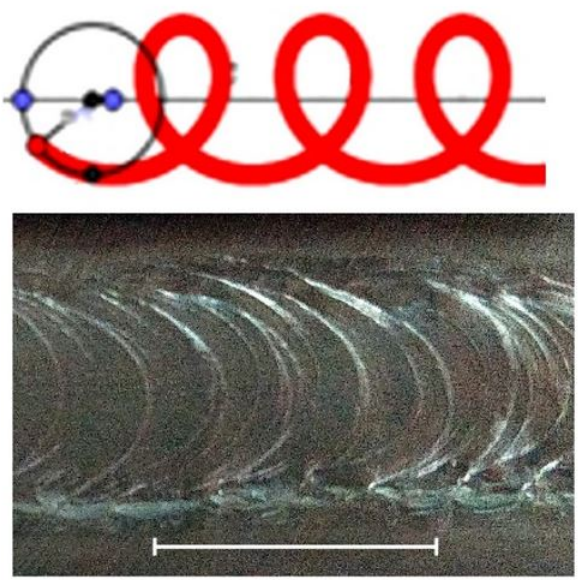

(b)

Figura 9. Cordões de soldagem obtidos com o processo GTAW autógeno e os padrões de tecimento (a) e (b) (Tabela 1). Sentido de soldagem: para a esquerda. A escala nas imagens corresponde a $10 \mathrm{~mm}$.

O padrão correspondente à condição (a) (Tabela 1 e Figura 8a), somado à translação da tocha de soldagem, resulta no movimento esquematizado na parte superior da Figura 9a. Considerando a velocidade de soldagem $(1,4 \mathrm{~mm} / \mathrm{s})$ e a frequência de oscilação transversal $(0,5 \mathrm{~Hz})$ usadas, obtém-se uma distância esperada de $2,8 \mathrm{~mm}$ entre duas escamas sucessivas do mesmo lado do cordão. Comparando-se esse valor com separação média entre as escamas de tecimento medida na superfície do cordão $(2,8 \pm 0,3 \mathrm{~mm})$, observa-se que a movimentação lateral do arco está sincronizada com o deslocamento linear da soldagem. O movimento resultante também sugere o retrocesso periódico do arco nas laterais do cordão, o que pode explicar o aspecto da superfície do cordão obtido que sugere a ocorrência de refusões periódicas nas laterais do cordão.

Na Figura 9b, o movimento resultante esperado está esquematizado na parte superior da mesma. Esse movimento resulta em um retrocesso periódico do arco, o que pode explicar as escamas observadas na superfície do cordão mostrado. Considerando, novamente, a velocidade de soldagem e a frequência de oscilação transversal usadas, pode-se prever uma

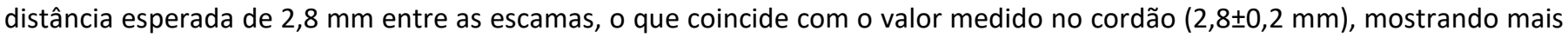
uma vez, que a movimentação do arco está sincronizada com o deslocamento linear da soldagem. A trajetória esperada do arco também indica que esse deve passar com uma maior velocidade do lado esquerdo do cordão (correspondente ao lado inferior na Figura 9b) e, periodicamente, deve permanecer relativamente estático do lado oposto. A maior velocidade de translação do arco no lado esquerdo do cordão poderia ser o fator que explicaria a presença eventual de mordeduras que foi observada com maior evidência desse lado do cordão.

Na Figura 10 são mostrados exemplos de cordões obtidos com o processo GMAW com transferência por curto-circuito. Uma primeira observação relevante é a pequena diferença de aparência dos cordões obtidos sem e com a oscilação magnética.

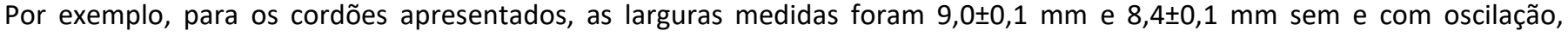
respectivamente. Pode-se observar, contudo, pequenos desvios na margem do cordão que foi feito com oscilação do arco. Embora seja difícil de caracterizar, medidas aproximadas obtidas com o Image $J$ indicam uma amplitude de deslocamento lateral do cordão de $0,3 \mathrm{~mm}$ a 0,4 $\mathrm{mm}$ ou cerca de $4 \%$ da largura total do cordão. Outro indício de atuação do campo magnético variável durante a soldagem são marcas de oxidação nas laterais do cordão em que o arco passou de forma mais intermitente (lateral superior da Figura 10b). Novamente, medidas com o Image J indicam uma separação média de aproximadamente $9,8 \mathrm{~mm}$ entre os centros dessas marcas. Considerando a velocidade de soldagem $(4,2 \mathrm{~mm} / \mathrm{s})$ e a frequência de oscilação $(0,5 \mathrm{~Hz})$ usadas no ensaio, a distância esperada entre os centros das marcas de oxidação seria de $8,4 \mathrm{~mm}$ considerando que essas estejam associadas com a oscilação do arco. Esta diferença entre o sincronismo da movimentação lateral do arco e o deslocamento linear da soldagem pode ser decorrente da pouca eficiência do sistema oscilador ao trabalhar com comprimento de arco variável (em média, pequeno). Possivelmente nem toda oscilação magnética imposta atuou na existência de um arco elétrico ou quando este estivesse com seu comprimento favorável para ser defletido. 

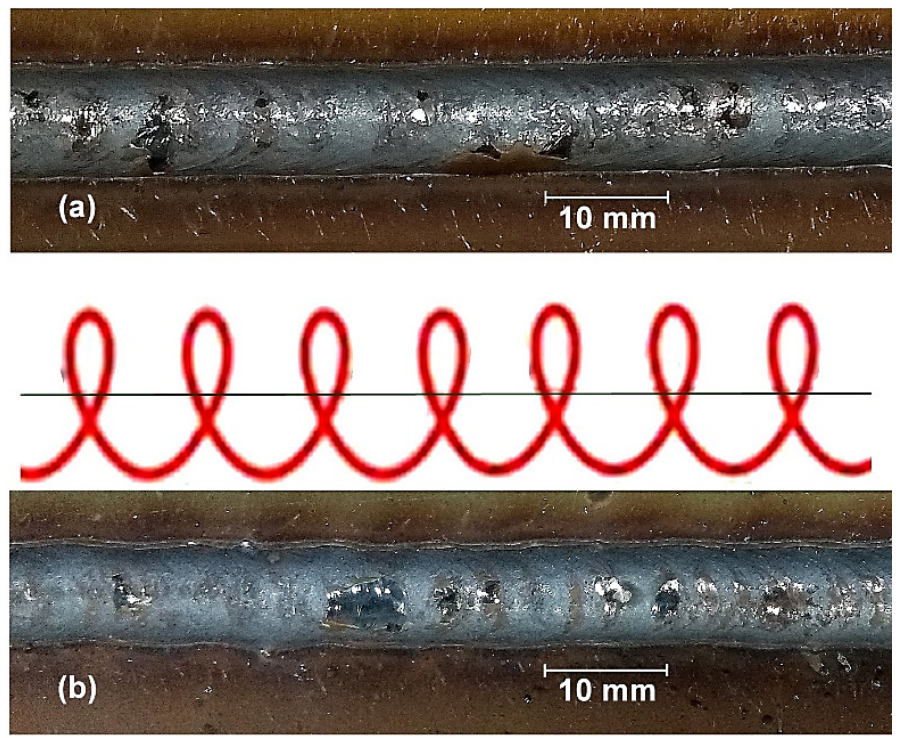

Figura 10. Cordões de soldagem obtidos com o processo GMAW transferência por curto-circuito. Em (a) sem oscilação e em (b) com oscilação junto com o padrão usado. Sentido de soldagem: Para a esquerda.

Esses resultados indicam um efeito muito menos intenso da aplicação do campo magnético durante soldagem GMAW com transferência por curto-circuito em comparação com a soldagem TIG. Outros possíveis motivos para esse menor efeito incluem o pequeno comprimento médio de arco típico da soldagem GMAW com transferência por curto-circuito e o maior valor da corrente de soldagem usada nesses testes. Adicionalmente, o maior volume da poça de fusão, que conta com a contribuição do arame fundido, pode levar a uma maior dificuldade da própria poça em acompanhar mudanças na direção do arco e resultar em menores alterações no formato do cordão de solda.

O aspecto dos cordões de solda obtidos com as condições (c) e (d) da Tabela 4 (GMAW pulsado) é mostrado na Figura 11 juntamente com um cordão produzido com os mesmos parâmetros (Tabela 3), mas sem a oscilação do arco. Comparando-se com os resultados obtidos com transferência por curto circuito (Figura 10), observa-se um maior efeito da oscilação magnética no formato do cordão, mesmo considerando a aplicação de menores campos magnéticos, o que possivelmente é explicado pelo maior comprimento de arco usado. Como esperado, observa-se também, que o efeito mais pronunciado do campo magnético ( $B x=11,6 \mathrm{mT}$ ) causou variações mais intensas no formato do cordão.

Em comparação com os resultados obtidos com a soldagem GTAW, os cordões obtidos com transferência pulsada também mostram uma menor influência da oscilação magnética apesar do uso de campos magnéticos muito mais intensos no segundo caso. Isso reforça o possível efeito de uma poça de fusão de maior volume em causar um amortecimento dos efeitos térmicos da movimentação do arco, reduzindo, assim, as variações no formato do cordão.

\section{Sem Oscilação}

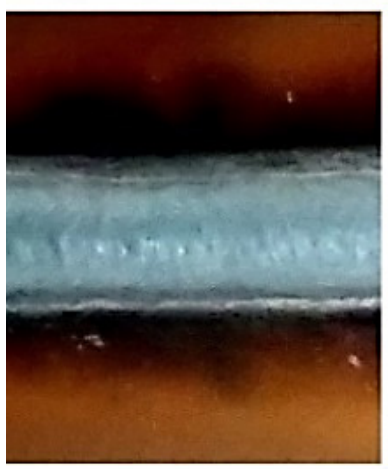

"Meia lua"
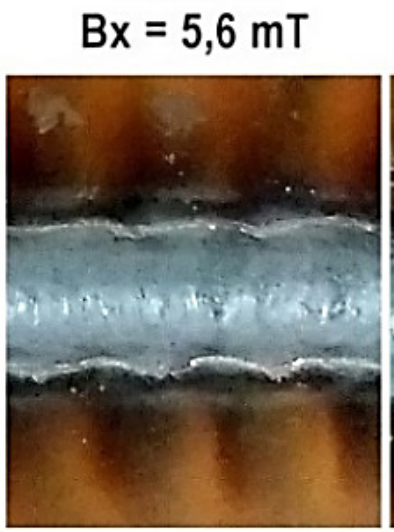

$10 \mathrm{~mm}$
"Meia lua" $B x=11,6 \mathrm{mT}$

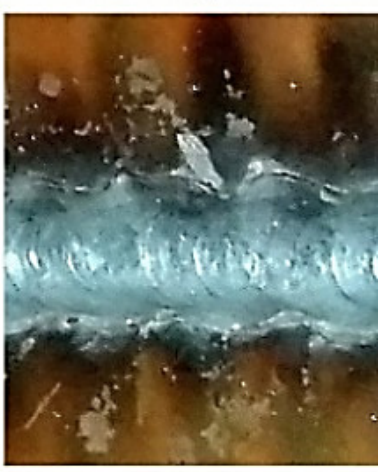

Figura 11. Cordões de solda obtidos com soldagem GMAW pulsada (Tabela 3) sem oscilação e com oscilação magnética do arco (condições (c) e (d) da Tabela 4). Frequência de oscilação 0,5Hz. 
Foi possível observar também que, a frequência de oscilação do campo magnético aplicado influencia, significativamente, o deslocamento lateral da poça de fusão, ver Figuras 12 e 13. Assim, enquanto o cordão obtido sem oscilação apresentou uma

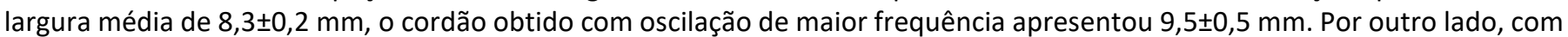
uma frequência de oscilação de $0,1 \mathrm{~Hz}$, embora o cordão tenha apresentado uma largura mais uniforme do que o obtido com maior frequência, a separação transversal entre os seus pontos de maior oscilação lateral foi de aproximadamente $11,3 \mathrm{~mm}$.

(a)

(b)

(c)

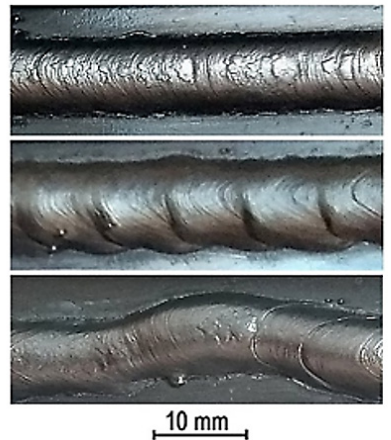

Sem oscilação

Oscilação: $0,5 \mathrm{~Hz}$

Oscilação: $0,1 \mathrm{~Hz}$

Figura 12. Cordões de solda obtidos com soldagem GMAW pulsada (Tabela 3) sem oscilação magnética do arco e com oscilação (condições (e) e (f) da Tabela 4).

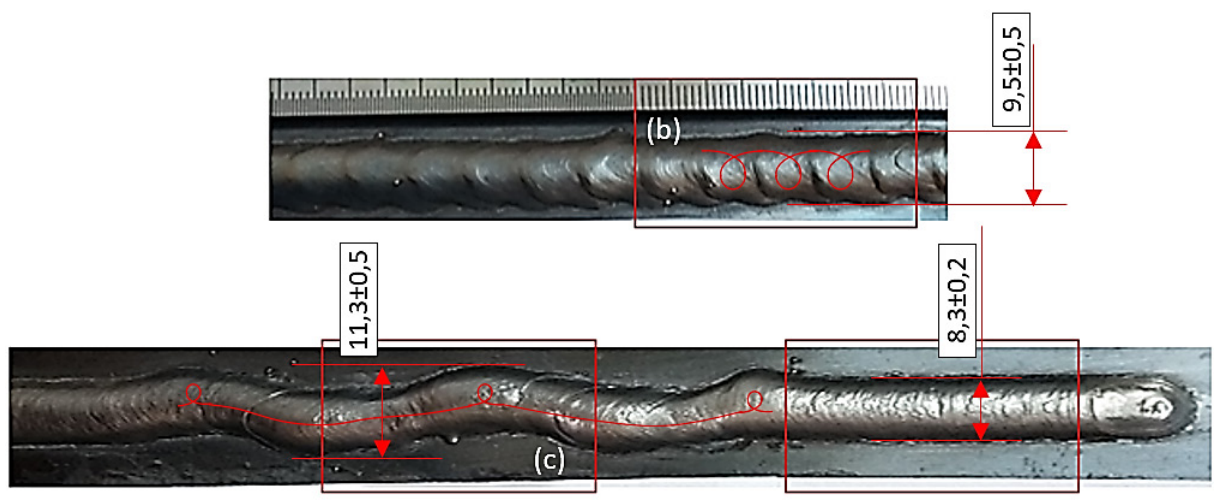

Figura 13. Cordões de solda obtidos com soldagem GMAW pulsada (Tabela 3) sem oscilação magnética do arco e com oscilação (condições (e) e (f) da Tabela 4). Figuras 13b e 13c correspondem a oscilações com 0,5 Hz e 0,1 Hz, respectivamente.

Na Figura 14, é apresentado o desvio de trajetória em um cordão obtido com a aplicação de oscilação transversal com uma onda "quadrada" com frequência de $0,1 \mathrm{~Hz}$ (condição (g) da Tabela 4). Como observado com o cordão obtido com a condição (f) e diferentemente do encontrado quando uma frequência mais elevada de oscilação é usada, observa-se um deslocamento lateral do cordão de aproximadamente $2,5 \mathrm{~mm}$ com a variação do campo magnético. Contudo, observa-se também que a mudança de posição do cordão ocorre de modo relativamente suave, ao longo de cerca de $7 \mathrm{~mm}$ (aproximadamente 1,8 s) de comprimento do cordão, apesar do campo magnético ter variado possivelmente em pouco milissegundos. Esse comportamento deve, também, estar ligado a uma maior inércia causada pelo maior volume da poça de fusão e/ou tempo de resposta do sistema quanto ao deslocamento de fluxo magnético em função da frequência aplicada.

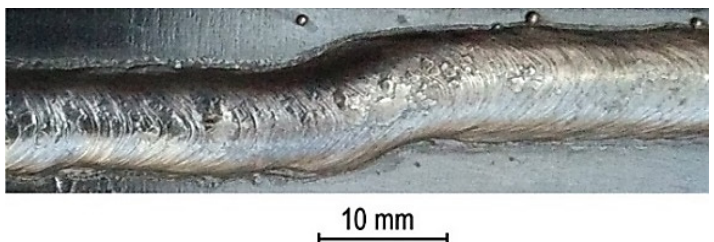

Figura 14. Cordão de solda obtidos com soldagem GMAW pulsada (Tabela 3) com oscilação (condição (g) da Tabela 4).

Na Figura 15 são mostradas as variações de tensão e corrente de soldagem em conjunto com as tensões de alimentação dos eletroímãs de oscilação transversal e longitudinal do arco. Um aspecto a ser destacado são as variações de tensão, particularmente o aumento das tensões de base que ocorrem aproximadamente quando a tensão de alimentação do eletroímã 
para a oscilação transversal do arco atinge os seus valores máximos (em valor absoluto). Foi observado que, com a aplicação de campos magnéticos mais intensos, as variações das tensões elétricas se tornam mais intensas e podem levar a extinções do arco. Esse aspecto do processo é tratado em outro trabalho submetido para publicação na Revista Soldagem \& Inspeção [23].

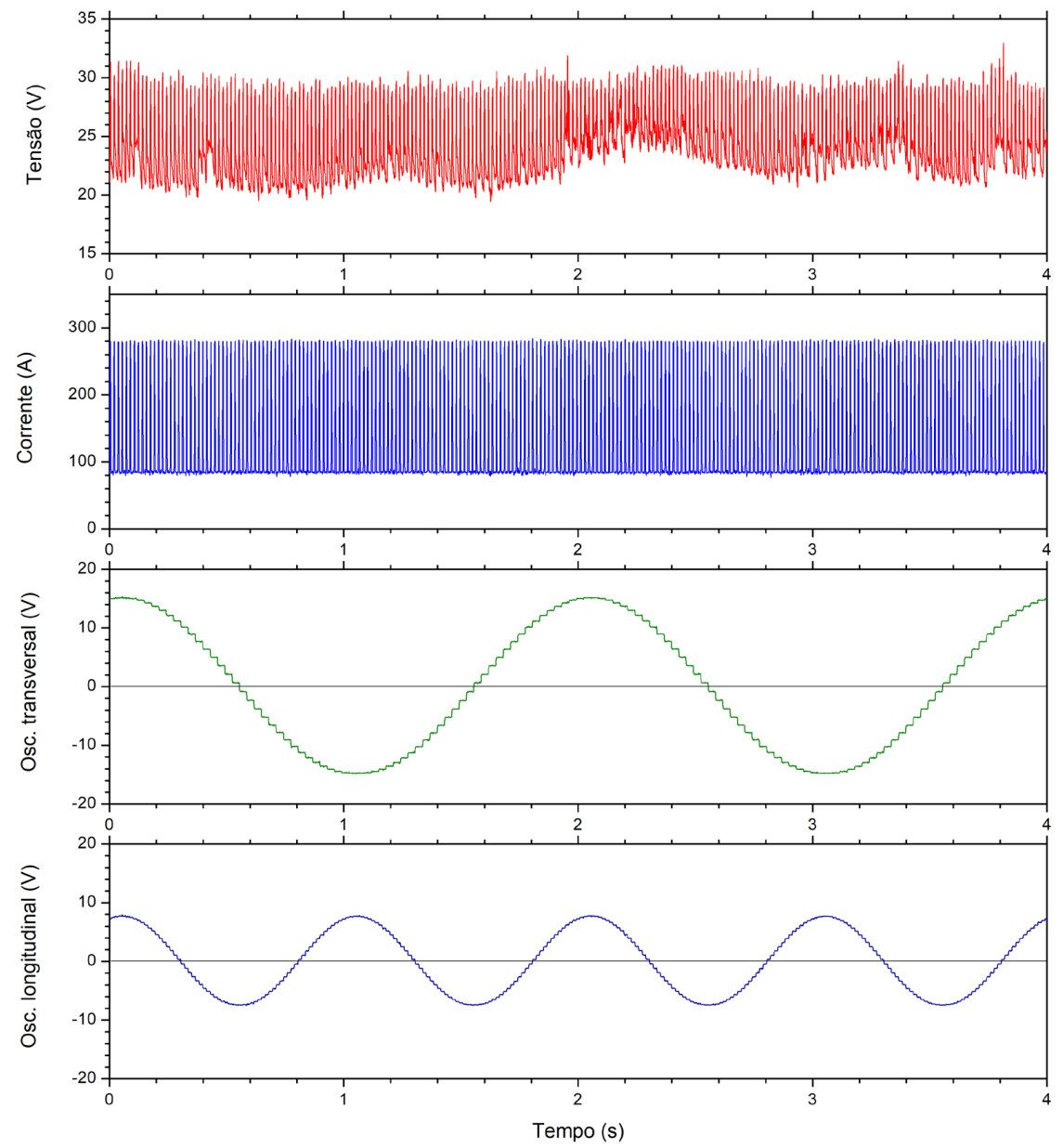

Figura 15. Parte dos oscilogramas de tensão e corrente de soldagem e variações das tensões de alimentação dos eletroímãs de um teste feito com os parâmetros indicados na Tabela 3 e a condição (c) da Tabela 4.

\section{Conclusão}

O presente trabalho descreve o desenvolvimento e a avaliação inicial de um sistema capaz de oscilar, de forma coordenada, o arco elétrico de soldagem tanto na direção transversal como na direção longitudinal ao eixo da solda. Foram feitos testes com os processos GTAW e GMAW e as conclusões obtidas foram:

- O sistema desenvolvido foi capaz de causar deslocamentos no arco elétrico coordenados nas direções transversal e longitudinal ao eixo de soldagem;

- $\quad$ O efeito dos campos magnéticos no formato do cordão foi mais pronunciado com o processo GTAW do que com o processo GMAW;

- No processo GMAW, o efeito da oscilação magnética do arco foi mais pronunciado em testes com transferência pulsada do que com transferência por curto circuito. Essa diferença foi associada principalmente ao maior comprimento médio do arco na operação com corrente pulsada;

- $\quad$ Para uma dada intensidade de campo magnético, nos testes de soldagem GMAW pulsada, a movimentação do arco foi dependente da frequência de aplicação do campo magnético.

\section{Agradecimentos}

Agradecimento especial ao Professor Elmo Salomão Alves do Departamento de Física da UFMG por disponibilizar o laboratório e equipamentos para as medições de campo magnético. 


\section{Referências}

[1] Mustafin FM, Blekherova NG, Kvyatkovskiy OP, Suvorov AF, Vasiliev GG, Gamburg IS, et al. Soldagem de tubulações. Moscow: Nedra; 2002. p. 65

[2] Akulov Al, Belchuk GA, Demyantsevich VP. Tecnologia de soldagem por fusão elétrica. Moscow: Machinostroenie; 1977. p. 21.

[3] Poluxin PI, Grikberg BG, Jadan VT, Cantenik SK, Vasiliev DI. Tecnologia dos materiais e soldagem. Moscow: Escola Superior MOSVA; 1977. p. 312.

[4] Polysoude SAS [internet]. 2012. [acesso em 15 fev. 2019]. Disponível em: http://www.polysoude.com/

[5] Serimax. Welding Technology Centre [internet]. 2019. [acesso em 15 fev. 2019]. Disponível em: http://www.serimax.com/en/about-uspremium-welding-solutions/

[6] Arc Products. Lincoln Electric Company. Controle de arco magnético DirectArc [internet]. 2019. [acesso em 8 out. 2019]. Disponível em: https://www.arcproducts.com/product/directarc-magnetic-arc-control/

[7] Hangil. Magnetic Arc Weave [internet]. 2019. [acesso em 8 out. 2019]. Disponível em: http://www.autowelding.com/eproduct29.htm

[8] CRC-Evans ${ }^{\circledast}$ [internet]. 2019. [acesso em 8 out. 2019]. Disponível em: http://www.crc-evans.com

[9] Reis RP, Souza D, Scotti A. Models to describe plasma jet, arc trajectory and arc blow formation in arc welding. Welding in the World. 2011;55(3-4):24-32. http://dx.doi.org/10.1007/BF03321283.

[10] Clain FM, Teixeira PRDF, Araújo DB. Two heat source models to simulate welding processes with magnetic deflection. Uberlândia: Universidade Federal de Uberlândia; 2017. http://dx.doi.org/10.1590/0104-9224/si2201.10.

[11] Juliani L. Utilização de um sistema de controle por oscilação magnética para a deposição de metal em passe de raiz utilizando o processo GMAW [dissertação]. Belo Horizonte: Universidade Federal de Minas Gerais; 2014.

[12] Kang YH, Na JS. Study on the modeling of magnetic arc deflection and dynamic analysis. Welding Journal. 2002;81(1):8-13.

[13] Li Y, Wu CS, Wang L, Gao JQ. Analysis of additional electromagnetic force for mitigating the humping bead in high-speed gas metal arc welding. Journal of Materials Processing Technology. 2016;229:207-215. http://dx.doi.org/10.1016/j.jmatprotec.2015.09.014.

[14] Passos TA. Efeito da oscilação magnética do arco na microestrutura e na geometria do cordão de revestimento de aço inoxidável depositados por soldagem TIG [dissertação]. Rio Grande: Universidade Federal do Rio Grande; 2016.

[15] Wang L, Wu C, Chen J, Gao J. Influence of the external magnetic field on fluid flow, temperature profile and humping bead in high speed gas metal arc welding. International Journal of Heat and Mass Transfer. 2018;116:1282-1291. http://dx.doi.org/10.1016/j.ijheatmasstransfer.2017.09.130.

[16] Wu H, Chang Y, Lu L, Bai J. Review on magnetically controlled arc welding process. International Journal of Advanced Manufacturing Technology. 2017;91(9-12):4263-4273. http://dx.doi.org/10.1007/s00170-017-0068-9.

[17] Bonacorso NG, Hubert IL, Cunha VP, Romero IA, Rosa GH, Kruger LC, et al. Desenvolvimento de um braço robótico portátil para a execução de soldagens automatizadas. Revista Técnico-Científica. 2014;1:1-13.

[18] Landgraf FJG. Propriedades magnéticas de aços para fins elétricos. São Paulo: Instituto de Pesquisas Tecnológicas do Estado de São Paulo; 2014.

[19] Universidade Federal de Santa Catarina. Materiais magnéticos: capítulo II. Florianópolis; 2014.

[20] Batistela NJ, Carlson R, Sadowski N, Leite JV. Caracterização magnética de lâminas de aço silício e avaliação das perdas no ferro sob vários regimes de indução. Revista Controle \& Automação. 2002;13(2):156-164. http://dx.doi.org/10.1590/S0103-17592002000200007.

[21] Sartori F. Tecnologias e técnicas modernas do processo MIG/MAG para o passe de raiz em soldagem orbital de tubos. Florianópolis: Universidade Federal de Santa Catarina; 2016.

[22] Universidade Federal do Rio de Janeiro. Capítulo 6: circuitos resistivos alimentados com onda senoidal: indutância mútua [internet]. 2020. [acesso em 14 jan. 2019]. Disponível em: http://fisexp3.if.ufrj.br/Roteiros/FisExp_3_Aula6.pdf

[23] Juliani L, Bracarense AQ, Medenesi PJ. Estudo do comportamento do arco elétrico GMAW pulsado sob efeito da oscilação magnética usando misturas de gás de proteção contendo dióxido de carbono. Belo Horizonte: Universidade Federal de Minas Gerais; 2020. 